University of Nebraska - Lincoln

DigitalCommons@University of Nebraska - Lincoln

1988

\title{
Computer Simulation of the Melting Process in Linear Macromolecules
}

Donald W. Noid

Oak Ridge National Laboratory

George A. Pfeffer

University of Nebraska at Omaha

Stephen Z. D. Cheng

University of Akron

Bernhard Wunderlich

University of Tennessee - Knoxville

Follow this and additional works at: https://digitalcommons.unl.edu/usdoepub

Part of the Bioresource and Agricultural Engineering Commons

Noid, Donald W.; Pfeffer, George A.; Cheng, Stephen Z. D.; and Wunderlich, Bernhard, "Computer Simulation of the Melting Process in Linear Macromolecules" (1988). US Department of Energy Publications. 40.

https://digitalcommons.unl.edu/usdoepub/40

This Article is brought to you for free and open access by the U.S. Department of Energy at DigitalCommons@University of Nebraska - Lincoln. It has been accepted for inclusion in US Department of Energy Publications by an authorized administrator of DigitalCommons@University of Nebraska - Lincoln. 


\title{
Computer Simulation of the Melting Process in Linear Macromolecules
}

\author{
Donald W. Noid, George A. Pfeffer, ${ }^{\dagger}$ Stephen Z. D. Cheng, ${ }^{*, \ddagger}$ and \\ Bernhard Wunderlich \\ Chemistry Division, Oak Ridge National Laboratory, Oak Ridge, Tennessee 37831, and \\ Department of Chemistry, The University of Tennessee, Knoxville, Tennessee 37996-1600. \\ Received January 13, 1988; Revised Manuscript Received April 29, 1988
}

\begin{abstract}
Polymer crystal melting kinetics is simulated by using molecular dynamics. A simplified molecular model of polyethylene crystal melting consisting of one extended chain on a polyethylene-like crystal surface is presented. The simulation data indicate that the end-to-end distance (eed) of radius of gyration (rg) has a simple exponential dependence on time. The rate constants for eed and $\mathrm{rg}$ are found to be temperature and molecular length dependent. A more detailed analysis of polymer crystal melting will be described.
\end{abstract}

\section{A. Introduction}

The melting kinetics of polymer crystals has been studied much less than crystallization kinetics. ${ }^{1}$ The main reason lies in the smallness of the crystals. Fringed micellar crystals and also chain-folded, lamellar crystals melt so fast that the limiting factor is often the conduction of heat to the interface. Reorganization and recrystallization, as well as relaxation of strained tie molecules, may further obscure the melting process.

Experimentally, slow melting leads to superheating of crystals before fusion is complete. ${ }^{2}$ Well-crystallized, extended-chain crystals of macromolecules melt slowly enough so that the melting kinetics can be followed via microscopy. The most well-known studies of polymer melting are for polyethylene (PE), ${ }^{3}$ selenium (Se), ${ }^{4,5}$ and poly(ethylene oxide) (PEO).6,7 Two possible types of melting kinetics have been observed. One shows a linear relationship between melting rate and superheating $\Delta T^{3}$ and the other a linear relationship between the logarithmic melting rate and $\Delta T^{4,6}$ where $\Delta T$ is the temperature difference between the controlled temperature and the equilibrium melting temperature, $T_{\mathrm{m}}{ }^{\circ}$. The former melting type is "continuous", while the latter appears to be "nucleation controlled".

The superheating of a crystal on melting is significantly different from the supercooling on crystallization. Typically, a plot covering both crystallization and melting rates of macromolecules has a sharp discontinuity at or in the vicinity of the equilibrium melting temperature, as shown in Figure 1. The discontinuity arises from the need of molecular nucleation on crystallization, opposed by the continuous melting. Even for nucleating-controlled melting, the nucleation barrier $(\Delta G)$ is usually small so that significant melting starts close to $T_{\mathrm{m}}{ }^{\circ}$, while on crystallization significant supercooling to a metastable melt is possible. The melting of equilibrium crystals must start from the upper and lower edges of the outer, lateral crystal surfaces and proceeds by peeling off layer after layer of molecular chains in a direction perpendicular to the chain direction. The melting of a single chain must start at a chain end or fold at the edge of the crystal, continuing down the lateral surface. ${ }^{1}$

Thermodynamic principles of the melting do not provide structural information. However, despite the lack of this knowledge, adding a limited mechanistic model may round out the picture of polymer crystal melting. In this paper

\footnotetext{
'Present address: Department of Chemistry, University of Nebraska, Omaha, NE 68182-0109.

† Present address: Institute and Department of Polymer Science, University of Akron, Akron, $\mathrm{OH} 44325$. Work performed partially when at Rensselaer Polytechnic Institute, Troy, NY.
}

we attempt to develop a more detailed picture of the crystal melting process on a molecular level with the help of computer simulation. A simplified molecular model of polymer crystal melting using the technique of molecular dynamics will be presented in the first two sections. Our results for the melting kinetics of a single, extended chain on this crystal surface will be presented in sections D and E. This paper is a first step toward detailed phase transition simulation.

\section{B. Molecular Model of Polymer Crystal Melting}

We assume that there is a polymer crystal surface with a corrugated shape as shown in Figure 2. Two levels can be observed on the surface: an upper and a lower one. A surface with these characteristics may correspond, for example, to the $(100)$ or $(010)$ crystal planes in $\mathrm{PE}^{3}$ and to the $(100),(140)$, or $(010)$ planes in PEO. ${ }^{6}$ The $c$-axis is parallel to the ridges and valleys of the surface (Figure 2). The size of this surface is considered to be infinite.

As part of the crystal, one chain with $n$ atoms, connected by covalent bonds, lies on the surface (Figure 2). Such a chain provides a new interface layer between the crystal and melt. The total surface area of the crystal, however, does not change by addition of subtraction of such a chain.

Furthermore, it is assumed that there are no defects within the crystal, on the crystal surface, and along the selected chain. Melting of the chain will become possible when the temperature is raised above the equilibrium melting temperature of the crystal, $T_{\mathrm{m}}{ }^{\circ}$. At that temperature the crystal growth and melting rates should be equal. At present, no nucleation events are included in the model.

The simulation was carried out on the IBM 3083 of Oak Ridge National Laboratory. The total running time for a typical 100-ps simulation of a 400-atom chain was $\sim 20$ h. Progress in programming has been made recently so that in the future these times can be greatly reduced (or the polymer chains increased accordingly). Full accounts of this will be given in the next progress report.

\section{Molecular Dynamics Method}

The first step in a molecular dynamics treatment is the development of the Hamiltonian for the model problem to be studied. For the case discussed in the previous section, we consider the Hamiltonian in eq 1

$$
\begin{aligned}
H= & \sum_{i=1}^{N} \frac{\bar{P}_{i}^{2}}{2 m_{i}}+\sum_{i=1}^{N-1} V_{\text {bond }}\left(r_{i, i+1}\right)+\sum_{i=1}^{N-2} V_{\text {bend }}\left(\theta_{i, i+1, i+2}\right)+ \\
& \sum_{i=1}^{N} \sum_{|j-i| \geq 3}^{N} V_{\text {nonbond }}\left(r_{i, j}\right)+\sum_{i=1}^{N} \sum_{\text {surface atoms } j} V_{\text {nonbond }}\left(r_{i, j}\right)
\end{aligned}
$$

where $i$ and $j$ are positive integers. The first term of the 


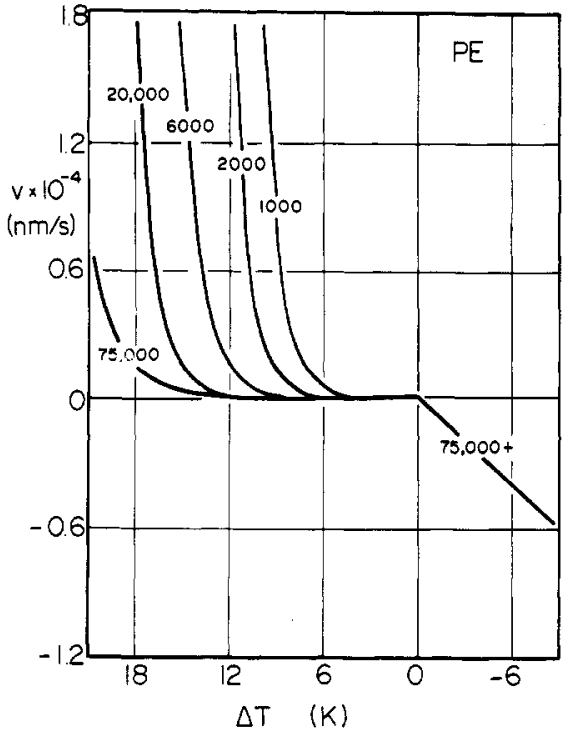

Figure 1. Melting and crystallization of polyethylene as a function of $\Delta T\left(\Delta T=T-T_{\mathrm{M}}\right)$. Crystallization expressed as linear crystal growth rate for various molecular masses. Melting measured on an extended chain crystal. For references, see: Wunderlich, B.; Cheng, S. Z. D. Gaz. Chim. Ital. 1986, 116, 345.

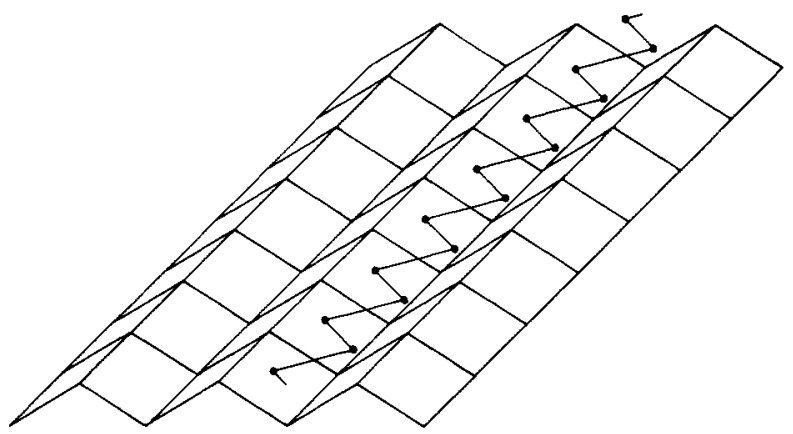

CHAIN ON CORRUGATED SURFACE

Figure 2. Crystal structure with a zigzag shape.

Hamiltonian represents the kinetic energy where the $\tilde{P}_{i}$ are Cartesian momenta of the $i$ particles and the $m_{i}$ the corresponding masses. In our study, we have collapsed the hydrogen atoms of the $\mathrm{CH}_{2}$ group to a single particle of mass 14.5, as also used by Weber ${ }^{7}$ in a model of the polyethylene chain. This is followed by four potential energy terms. The first of these represents a $\mathrm{C}-\mathrm{C}$ twobody, bonded harmonic oscillator function:

$$
V_{\text {bond }}(r)=1 / 2 K_{\mathrm{b}}\left(r-r_{\mathrm{e}}\right)^{2}
$$

where $r-r_{\mathrm{a}}$ is the deviation from the equilibrium distance between atoms $i$ and $j$ and $K_{\mathrm{b}}$ the force constant. The second potential energy term represents a bending, three-body interaction. We use a potential function as shown in eq 3 for its description:

$$
V_{\text {bend }}(\theta)=1 / 2 k_{\theta}\left(\cos \theta_{0}-\cos \theta\right)^{2}
$$

where $\theta$ is the angle between three bonded atoms in the chain and $k_{\theta}$ the corresponding force constant. The polymer is assumed to be completely flexible in this simulation since there are no four-body torsional potentials included. All interactions between nonbonded atoms are, however, included with a Lennard-Jones potential shown in eq 4 (third potential energy term):

$$
V_{\text {nonbond }}(r)=2 \epsilon\left[\left(r^{*} / r\right)^{9}-3 / 2\left(r^{*} / r\right)^{6}\right]
$$

where $r^{*}$ and $\epsilon$ represent constants fit to hydrocarbon

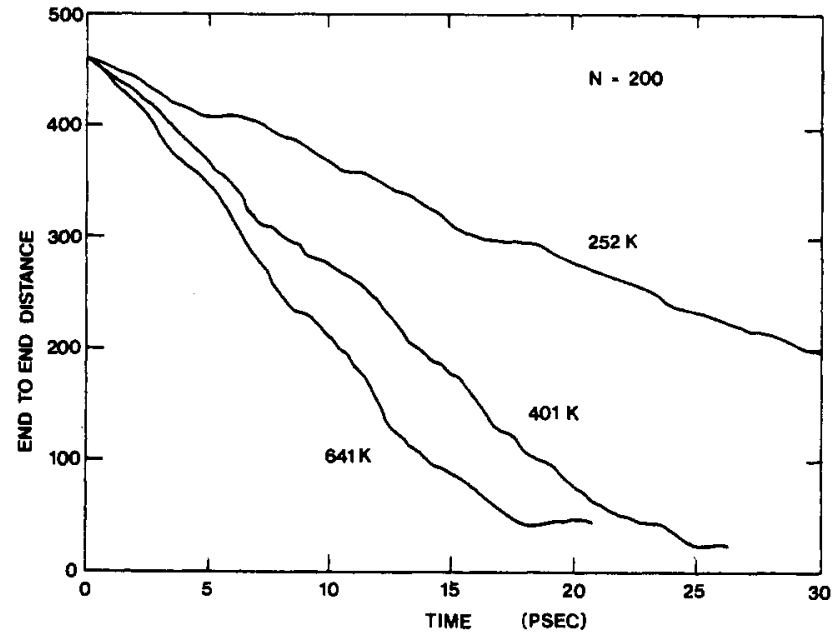

Figure 3. Computer simulation data of relationship between end-to-end distance and time at different temperatures for $N=$ 200.

Table I

Parameters for the Hamiltonian ${ }^{a}$

\begin{tabular}{ll}
\hline$V_{\text {bond }}(r)$ & $K_{\mathrm{b}}=2.466 \times 10^{-2}$ hartrees \\
$V_{\text {nonbond }}(r)$ & $\epsilon=1.90 \times 10^{4}$ hartrees \\
& $r^{*}=7.559 a_{0}$ \\
$V_{\text {bend }}$ & $k_{\theta}=4.951 \times 10^{-2}$ hartrees \\
& $\theta_{0}=113.3^{\circ}$
\end{tabular}

${ }^{a} a_{0}=0.0529 \AA$

interactions by Weber and $r$ is the separation distance. The nonbonded term was also used to model the surface interaction with the absorbed chain, the last term in eq 1. The surface is assumed to consist of $\mathrm{CH}_{2}$ groups in the center of each block of the corrugated grid. The constants used in eq 2-4 are presented in Table I. Hamilton's equations (eq 5) were integrated to generate the time dependence of momentum $P_{i}$ and the Cartesian conjugate position $\tilde{q}_{i}$ of the $i$ th atom in the chain in the crystallized configuration

$$
\partial H / \partial \tilde{q}_{i}=-\tilde{P}_{i} ; \quad \partial H / \partial \tilde{P}_{i}=\tilde{q}_{i}
$$

by using the differential equation solver ODE. ${ }^{8}$ The obtained results generally conserved the total energy within four to five digits. The initial conditions for the chain were chosen so as to put an extended polyethylene chain on the corrugated surface with the initial kinetic energy randomly distributed to create a desired temperature and to have no initial center of mass velocity. From the resulting trajectory, various time-dependent properties were computed and are discussed in the next section.

The initial simulation program of this molecular dynamics method reported on here had to use long computation times because of the large number of calculations in computing the two- and three-body forces in internal coordinates. Recent developments indicate that we will be able to make drastic reductions of the computing time by a new method of programming the chain rule derivatives of the internal coordinates.

\section{Simulation Results}

From the trajectory generated, we computed the endto-end distance (eed) and radius of gyration ( $\mathrm{rg}$ ) of the molecular chain as a function of time $(t)$. For the initial conditions $(t=0)$, the end-to-end distance is the same as the chain length since the chain was assumed extended on the crystal surface as shown in Figure 2. When melting starts under isothermal conditions, eed and $\mathrm{rg}$ of the chain 


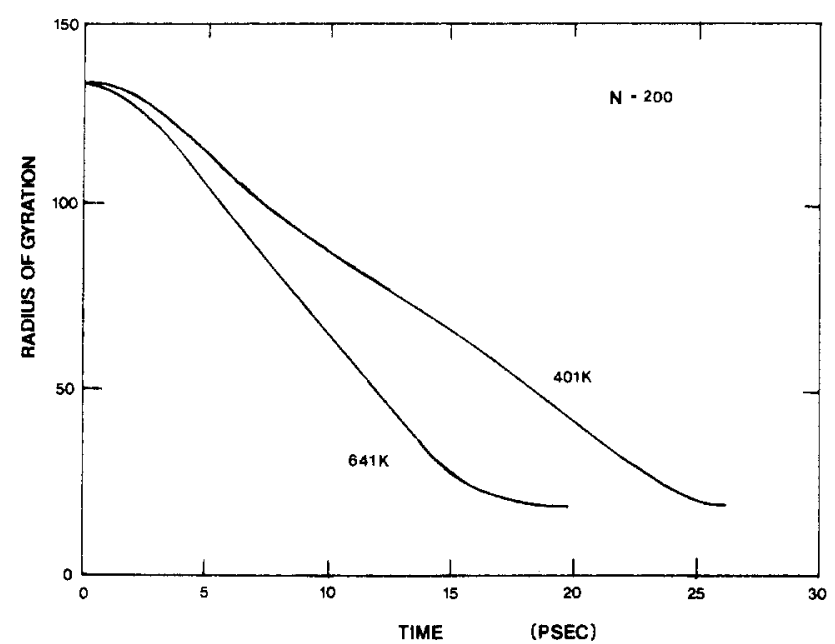

Figure 4. Computer simulation data of relationship between radius of gyration and time at different temperatures for $N=$ 200.

decrease with increasing time as shown in Figures 3 and 4 for $N=200$. Simultaneously, the chain gradually leaves the crystal surface to become more or less a coiled structure (with excluded volume effect). It approaches the "selfavoiding walk" in a limited three-dimensional space (with a wall formed by the crystal surface). If the time of computer simulation is long enough, the values of eed and $\mathrm{rg}$ reach a constant value, about $50 a_{0}$ for the end-to-end distance and $20 a_{0}$ for the radius of gyration. The corresponding ratio of the mean square values for the selfavoiding walk chain in three-dimensional space is $6.4^{9}$ and is in good agreement with our observed value of $\sim 6.25$. Furthermore, such a melting process is highly temperature and molecular length $(N)$ dependent. A faster melting process occurs at a shorter molecular length and at a higher melting temperature. The molecular length dependence of the melting is coupled to lamellar crystal thickness since we are dealing with the melting process of an extended chain crystal.

Quantitatively, the simulation data can be cast into a exponential function

$$
\text { eed }=\operatorname{eed}_{0} \exp \left(-\lambda_{\text {eed }} t\right)
$$

where eed $\mathrm{d}_{0}$ is the initial end-to-end distance (in our case the length of extended chain), $t$ is the time, and $\lambda_{\text {eed }}$ is the rate constant that indicates how fast the melting process proceeds. The rate constant $\lambda_{\text {eed }}$ was found by computing the least-squares fit of the slope of $\ln \operatorname{eed}(t)$ vs $t$. The data of $\ln$ eed $(t)$ vs $t$ were fit over the range of $t$ from 0 to 10 ps by using the Harwell program VCO4AD, which is a standard least-squares fit of a straight line to data. For all data sets considered, we found excellent reliability of our fit and model. A similar result was computed from the simulation data of radius of gyration, $R G$, but since it does not provide any independent information on the melting process, it will not be considered further.

The temperature and molecular length dependences of the rate constant $\lambda_{\text {eed }}$ are shown in Figures 5 and 6 . From Figure 5 a linear relationship between $\ln \lambda_{\text {eed }}$ and $1 / T$ can be found. The slope indicates a single activation energy for $\lambda_{\text {eed }}$, and the intercept of $1 / T=0$ is the value of the preexponential term. For the case of $N=100$ a limiting rate is computed which indicates a different type of process may be occurring. The molecular length (or lamellar thickness) dependence is shown in Figure 6. We can see a linear relationship between $\ln \lambda_{\text {eed }}$ and $1 / N$. A similar interpretation of the slope and intersection can be obtained.

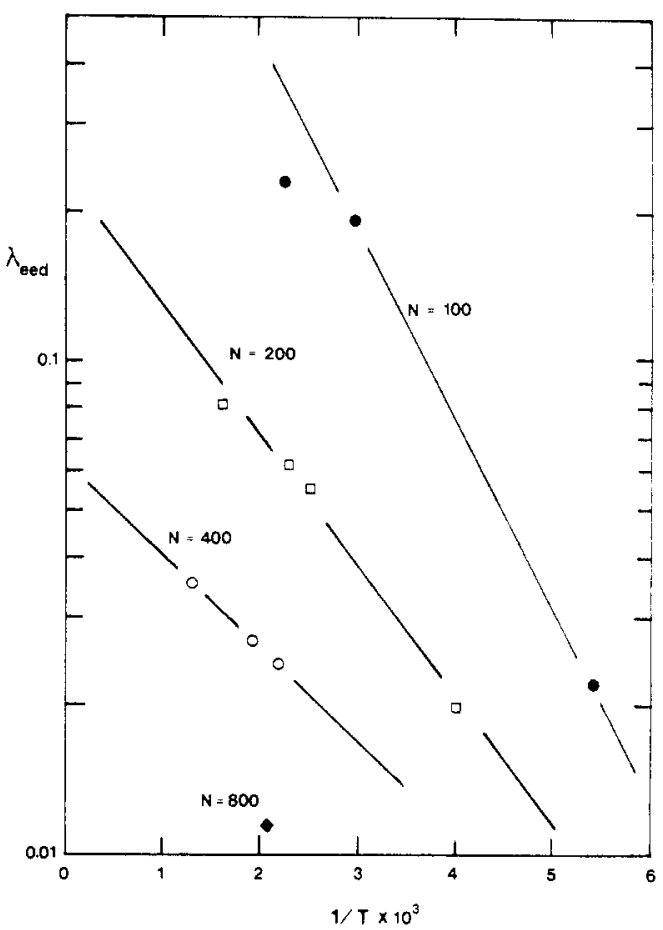

Figure 5. Relationship between $\lambda_{\text {ad }}$ and $1 / T$ for different molecular lengths $(n=100,200,400$, and 800$)$.

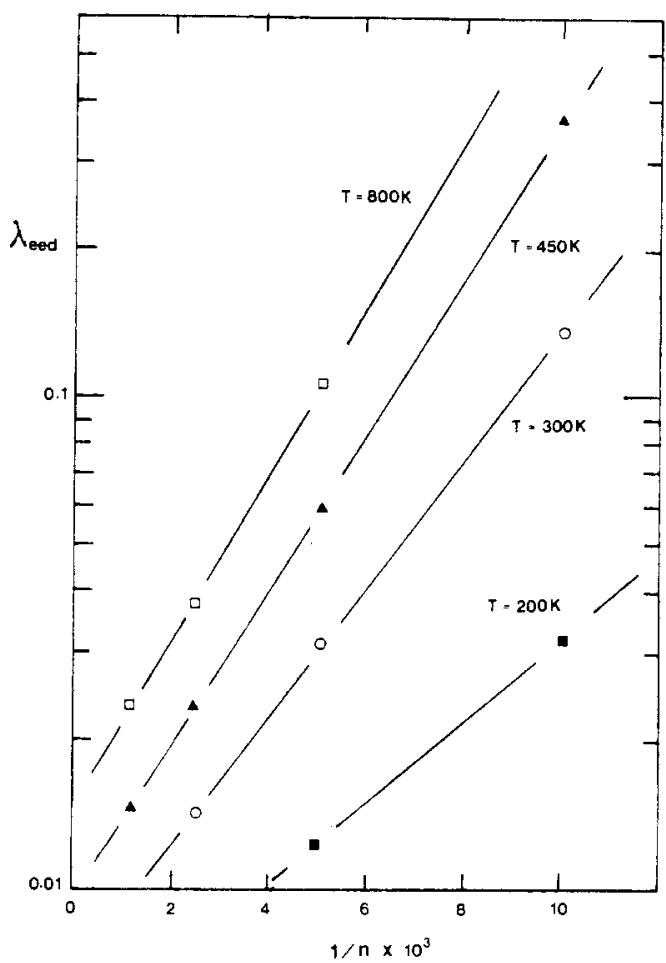

Figure 6. Relationship between $\lambda_{\text {and }}$ andecular length at different temperatures ( $T=200,300,450$, and $800 \mathrm{~K})$.

Videotapes of the simulation process have been made and can be viewed on request.

\section{E. Discussion}

The melting process of one extended chain on the zigzag crystal surface has been studied with the help of computer simulation. Dynamic changes of the end-to-end distance and radius of gyration have been found. We have introduced a new parameter, $\lambda$, to describe the rate of the melting process. Temperature and molecular length (or lamellar thickness) dependences of $\lambda$ are found for both 
end-to-end distance and radius of gyration.

To find the relationship between the simulation data studied here and macroscopic experimental observation of the melting rate, one must assume that the melting process occurs one layer after the other and also that any one extended chain molecule is totally molten as soon as its end-to-end distance (or radius of gyration) reaches the random coil value in the three-dimensional melt. The melting rate observed experimentally can thus be connected with the rate constant $\lambda_{\text {eed }}$ or $\lambda_{\text {g. }}$. Based on eq 6 therefore, the melting process simulated here could be called "nucleation-controlled". However, in our computer simulation only one extended chain, rather than a layer of extended chains, was taken into account. The price of such a simplification is thus paid by a decrease of interactions between the selected chain and its neighbors (as given by eq 4). Therefore, the melting rate in our simulated data should be considerably speeded up.

Assuming that the rate of the melting process could be described by the simulated data here, typical experiments are 4-5 orders of magnitude slower. By use of data on the melting kinetics of extended chain PE crystals, melting rates of $0.1-1 \mu \mathrm{m} / \mathrm{s}$ have been detected. ${ }^{10}$

Three reasons may account for this discrepancy. The first one has already been detailed above; i.e., our simulated data are for one extended chain only, not for one layer, and even less for observable macroscopic crystal thicknesses. The second may be that the coefficients of the interaction in eq 1-4 were not properly chosen. They may cause too few or too small an interaction between the chain and the simulated crystal surface. This is reflected also in the fact that melting as simulated occurs even at temperatures lower than the equilibrium melting temperatures $\left(T_{\mathrm{m}}{ }^{\circ}=\right.$ $414.3(N-1.5) /(N+5.0)$, where $N$ is the number of $\mathrm{CH}_{2}$ groups). ${ }^{1}$ The third reason may be that there is a pause after completion of the melting of the first layer until the second layer is molten; i.e., one may assume one must first produce "molten nuclei" on each layer. Overall, the first two reasons are very likely; the third is more speculative.

Further work is thus necessary in two directions: first, to search for the relationship between $\lambda$ and crystal melting rates, and second, to extend our simulation to multiple chain systems and introduce defects on the crystal surface in order to fully understand polymer crystal melting in a molecular level.

Acknowledgment. This work has been sponsored by the Division of Materials Sciences, Office of Basic Energy Sciences, U.S. Department of Energy, under contract DE-AC05-840R21400 with Martin Marietta Energy Systems, Inc., and the Polymers Program of the National Science Foundation, Grant DMR 8317097. The final writing and discussion of this paper were supported in part by the Institute and Department of Polymer Science, The University of Akron.

Registry No. Polyethylene, 9002-88-4.

\section{References and Notes}

(1) Wunderlich, B. Macromolecular Physics; Academic: New York, 1980; Vol. III.

(2) Hellmuth, E.; Wunderlich, B. J. Appl. Phys. 1965, 36, 3039; Hellmuth, E.; Wunderlich, B.; Rankin, J. M. Appl. Polym. Symp. 1966, 2, 101. Jaffe, M.; Wunderlich, B. In Thermal Analysis; Schwenker, E. F., Garn, P. D., Eds; Academic: New York, 1969; Vol. 1, p 387.

(3) Czornyj, G.; Wunderlich, B. J. Polym. Sci., Polym. Phys. Ed. 1977, 15, 1905. Wunderlich, B. Faraday Discuss. Chem. Soc. 1979, No. 68, 237.

(4) Shu, P. H.-C.; Wunderlich, B. J. Cryst. Growth 1980, 48, 227.

(5) Crystal, A. H. J. Polym. Sci., Polym. Phys. Ed. 1970, 8, 2153.

(6) Kovacs, A. J.; Gonthier, A. Kolloid Z. Z. Polym. 1972, 250, 530. Kovacs, A. J.; Gonthier, A.; Straupe, C. J. Polym. Sci., Polym. Symp. 1977, 50, 283; 1977, 59, 317.

(7) Weber, T. A. J. Chem. Phys. 1978, 69, 2347.

(8) Shampine, L. F.; Gordon, M. K. Computer Solutions of Ordinary Differential Equations: The Initial Value Problem; Freeman: San Francisco, 1975.

(9) Barr, R.; Brender, C.; Lax, M. J. Chem. Phys. 1981, 75, 453.

(10) Czornyj, G.; Wunderlich, B. J. Polym. Sci., Polym. Phys. Ed. $1977,15,1905$. 